\title{
RESULTS WITH THE EXTENDED MERLIN
}

B. Anderson \& R.D. Davies

Nuffield Radio Astronomy Laboratories, Jodrell Bank, Macclesfield, Cheshire SK11 9DL, UK

\section{Introduction}

A major enhancement of MERLIN completed in 1992 has led to a transformation of its capabilities. The seventh telescope added to the array is a $32-\mathrm{m}$ telescope at Cambridge which provides high sensitivity on all the longest baselines where extra collecting area is especially beneficial. The $32-\mathrm{m}$ telescope is a shaped primary-Cassegrain configuration which achieves an 80 percent aperture efficiency. A change from frequency to amplitude modulation in the microwave links increased the effective bandwidth from 9 to $30 \mathrm{MHz}$. This broadbanding enables the two hands of circular polarization to be transmitted simultaneously, each in a $15 \mathrm{MHz}$ bandwidth. A further improvement in the system sensitivity was achieved by cryogenically cooling the low noise amplifiers in the front-end receivers.

The central processing correlator was also upgraded during this time. Two-bit has replaced one-bit sampling thereby giving a direct 37 percent improvement in sensitivity. 4600 physical channels, capable of being fed from 13 telescope inputs, are available at the maximum bandwidth of $15 \mathrm{MHz}$; data recirculation is used to double the number of channels and quadruple the resolution for each halving of the input bandwidth - a facility of benefit in the spectral line observing mode.

Preliminary imaging with MERLIN can now be performed through an automatic pipeline system which provides a dynamic range of up to $100: 1$, sufficient for weaker sources. When the strength of the source allows, higher fidelity images can be produced by more sophisticated techniques. It is now possible to use multiband mapping within the 10-15 per cent bandwidths available at 1.6 and $5 \mathrm{GHz}$ and improve image fidelity even further. Polarization can be measured to an accuracy of 1 percent.

\section{The Performance of MERLIN}

During 1992 MERLIN has operated at a frequency of $5 \mathrm{GHz}$ where 6 telescopes are available (the MkIII telescope at Wardle has low efficiency at this frequency). The theoretical resolution of 50 mas is achieved as is the theoretical noise performance of 70 $\mu \mathrm{Jy}$ rms per beam area in $12 \mathrm{hr}$ of observation. 
Earlier observations with MERLIN (as with VLBI) relied heavily on the phase closure property which restricted most observations to sources which were detectable in one integration (a few minutes). This approach is now largely superseded by the phase-referencing technique which observes a reference source in a cyclic sequence with the target source. It is found that at 5 $\mathrm{GHz}$ the reference source must be closer than $3^{\circ}-5^{\circ}$ in order for the atmospheric/ionospheric irregularities to be compensated. Sources as weak as several $\mathrm{mJy}$ have been mapped at the theoretical resolution and sensitivity. The first phase of a programme to identify reference sources has produced 800 compact sources in the Dec $=35^{\circ}-75^{\circ}$ range with an rms position accuracy of 12 mas. (Patnaik et al. 1992a). An important feature in the operation of MERLIN is the reliability of all its components telescopes, microwave links, L-band links, cryogenic receivers and the correlator which all have to work simultaneously. The current efficiency of operation is 80 percent.

\section{Astronomy}

The resolution produced by its $230 \mathrm{~km}$ maximum baseline and its rms sensitivity of 50-100 $\mu \mathrm{Jy}$ in 12 hours of observation, make MERLIN an ideal observing system for a wide range of astronomical programmes. One of the first programmes on the new MERLIN at $5 \mathrm{GHz}$ was a study of gravitational lenses. These had previously been thought to be preferentially larger than 1 arcsec in size; MERLIN is making fertile discoveries on smaller angular scales, focussing on the detailed geometry of these objects to obtain the mass of the lensing objects and the value of Hubble's constant. (Patnaik et al. 1992b,c).

The high sensitivity of MERLIN at $5 \mathrm{GHz}$ has added a new stimulus to the study of stars in the radio continuum where its rms brightness temperature sensitivity is $700 \mathrm{~K}$. The largest stars can be resolved and the structure in binary and active stars can be mapped. As an example, the classical Nova Cygni 92 was resolved by MERLIN only 80 days after outburst (Pavelin et al. 1992). $\mathrm{OH}$ and $\mathrm{H}_{2} \mathrm{O}$ maser observations are also an obvious use of MERLIN.

A wide range of AGNs have been mapped by the new MERLIN. Its high sensitivity is ideal for investigating the weaker emission from Seyfert and star-burst galaxies where it also has a role in distinguishing the free-free from synchrotron emission as in NGC 4151 (Pedlar et al. 1992). The new MERLIN will make a 
significant contribution to astrometry. Absolute positions can be determined to better than 10 milliarcsec and relative positions to better than 1 millarcsec. MERLIN has demonstrated a unique astrometric capability in the studies of pulsar proper motions.

\section{The Availability of MERLIN}

MERLIN is a National Facility available to all astronomers through the MERLIN Time Allocation Committee of the SERC PATT system on application to the Director NRAL. The frequency available during 1993 will be L-band ( 1.3 to $1.7 \mathrm{GHz})$ followed by $22 \mathrm{GHz}$ in early 1994 .

Many programmes benefit from a combination of arrays. EVN combined with MERLIN produces very sensitive maps on baselines of $5 \mathrm{~km}$ to $1000 \mathrm{~km}$. It should be remembered that MERLIN in its present mode of operation is fixed in frequency for about one year at a time so VLBI observations which require MERLIN are only available on timescales of about 2 years; it is hoped to provide more frequency flexibility in the next few years.

\section{References}

Patnaik, A.R. et al. 1992a. M.N.R.A.S., 254, 665.

Patnaik, A.R. et al. 1992b. M.N.R.A.S., 259, 1P.

Patnaik, A.R. et al. 1992c. M.N.R.A.S., in press.

Pavelin, P.E. et al. 1992. Nature, submitted,

Pedlar, A. et al. 1992. M.N.R.A.S. submitted. 